\title{
Recovery of waste heat from engines: an $A D$ view
}

\author{
Miguel Cavique ${ }^{1, *}$, João Fradinho ${ }^{2}$, António Gabriel-Santos ${ }^{2}$, António Mourão $^{2}$ and António Gonçalves-Coelho ${ }^{2}$ \\ ${ }^{1}$ UNIDEMI, CINAV \& Escola Naval, Base Naval de Lisboa - Alfeite, 2810-001 Almada, Portugal \\ ${ }^{2}$ UNIDEMI \& DEMI, Faculdade de Ciências e Tecnologia, Universidade Nova de Lisboa, Campus de Caparica, 2829-516 Caparica, \\ Portugal
}

\begin{abstract}
The Newcomen engine (1705) and the Watt engine (1769) are good examples of coupled and uncoupled designs. The Watt engine had an efficiency of about 3\%, a shallow value when compared to engines of nowadays while resulting in a significant increase at those times. According to Axiomatic Design, Watt engine had a better performance than the Newcomen design due to its uncoupled nature. This work aims at applying the same reasoning to choose between new inventions designed for recovering waste heat from engines as to produce work. The most popular of those inventions are the organic Rankine cycle (ORC) and the Kalina cycle. Marine applications use those inventions to improve the efficiency of Diesel engines because the increase of weight of the recovery system does not affect power propulsion. A controversial regarding what system to develop occurred in scientific community as well as in the industry. The application of AD to those cycles classifies ORC as an uncoupled design and the Kalina cycle as a coupled design. Therefore, the ORC might be pondered for future development regarding energy efficiency.
\end{abstract}

\section{Introduction}

The conference of Paris (2015) organized by the United Nations ended with a settlement between the vast majority of the World countries regarding the reduction of the emissions of greenhouse gases (GHG). The World States were in accordance to reduce their carbon emissions "as soon as possible" and to do their best to keep global warming "well below $2{ }^{\circ} \mathrm{C}$ " of the preindustrial level.

With this in mind, the EU goals for 2030 include achieving at least $27 \%$ energy savings when compared with the business-as-usual scenario. The most effective way of fulfilling this target is to increase the efficiency of energy production. The energy sector of the EU28 reports the production of electricity and transportation as responsible for $82 \%$ of the GHG emissions [1]. The transport sector accounts for $33 \%$ of the final energy use in the EU28, making this sector a target for the increase in energy efficiency. The EU policy on energy aims to develop new technologies to "ensure secure, affordable and climate-friendly energy" [2]. Therefore, a new market of new technologies for waste heat recovery (WHR) appeared in the EU, formerly for large industrial plants and recently for plants with shaft power in the order of magnitude of $1 \mathrm{MW}$.

The WHR technology for marine diesel applications can increase the efficiency of the engines with a tonnage surplus of $0.5 \%$, which is insignificant regarding the required propeller power.

The most common WHR technologies for Diesel engines are the conventional steam Rankine cycle (SRC), the organic Rankine cycle (ORC) and the Kalina cycle (KC) [3]. All of these technologies can recover energy of waste heat of medium quality, ranging from $230{ }^{\circ} \mathrm{C}$ to $650{ }^{\circ} \mathrm{C}$. SRC is a well-known and proven technology that can be adapted to work with medium quality waste heat. The ORC, developed in the sixties, performs a Rankine cycle similar to the SRC using a refrigerant as working fluid and can be seen as a reverse refrigeration cycle. Finally, the $\mathrm{KC}$, named after its inventor, Alexander Kalina, in 1983, is the reverse of the absorption ammonia-water cycle.

At nominal load conditions, the temperature of the exhaust gas of the Diesel engines lies in the range of 300 ${ }^{\circ} \mathrm{C}$ to $500{ }^{\circ} \mathrm{C}$. Large Diesel engines have shaft efficiency near $50 \%$ and an energy waste of about $25 \%$ to $30 \%$ in the exhaust gas. The WHR technologies, with efficiencies of about $20 \%$, can increase the overall efficiency by about $10-15 \%$. Dig Vijay Singh, et al. [3] discussed the overall increase in efficiency of Diesel engines, concluding that SRC allows increasing the efficiency in the range of $2-7 \%$, ORC by $8-15 \%$ and $\mathrm{KC}$ in a similar range of ORC. SRC has the advantage of using a well-known technology, but the efficiency is moderate when compared to ORC or KC. Therefore, a controversy aroused in the industry regarding the best system, ORC or $\mathrm{KC}$, for recovering medium range waste heat from Diesel engines.

Ulrik Larsene et al. [4] modeled the recovery of energy from a marine two-stroke low speed engine with ideal SRC, as well as ORC using R245ca as working fluid, and KC. They found likely to achieve 5\% additional power using SRC, a similar value for $\mathrm{KC}$, and $7 \%$ additional power with ORC. However, at similar conditions, the maximum pressure of $\mathrm{KC}$ exceeds the

* Corresponding author: cavique.santos@marinha.pt 
high pressure of an ORC system. Paola Bombarda et al. [5] studied both systems applied to a truck Diesel engine that exhausts gas at $346{ }^{\circ} \mathrm{C}$. The $\mathrm{KC}$ needs a maximum pressure of 100 bar while ORC using hexamethyldisiloxane needs 9.7 bar to achieve a similar efficiency. In cogeneration plants, the exhaust gas temperature is about $150{ }^{\circ} \mathrm{C}$ making the efficiency and pressure lower than on the previous examples [6]. Diedie Lin et al. [7] compared ORC with R245fa to $\mathrm{KC}$ with different concentrations of ammonia, as well as with a SRC-ammonia. They modelled the systems and varied the hot water temperature of the heat source from $100{ }^{\circ} \mathrm{C}$ to $200{ }^{\circ} \mathrm{C}$, as to evaluate the sensibility of the efficiency concerning hot temperature. Regarding efficiency, ORC is better than any $\mathrm{KC}$ from $100{ }^{\circ} \mathrm{C}$ to $140{ }^{\circ} \mathrm{C}$, slightly worst than $\mathrm{KC}$ with high ammonia concentration for higher values of temperature. In the range $140{ }^{\circ} \mathrm{C}$ to 200 ${ }^{\circ} \mathrm{C}$, SRC-ammonia has the best efficiency of all systems under comparison [7].

This paper aims at using Axiomatic Design (AD) to choose between ORC and $\mathrm{KC}$ in what concerns to the recovery of energy from the exhaust gas of Diesel engines. Section 2 presents the simplest systems, ORC and $\mathrm{KC}$, and section 3 evaluates these cycles using AD. On section 4 and 5 the paper concludes that $\mathrm{KC}$ is a coupled design while ORC is an uncoupled design.

\section{Organic Rankine and Kalina Cycles}

$\mathrm{ORC}$ and $\mathrm{KC}$ are reverse cycles of the vaporcompression refrigeration and the ammonia-water absorption refrigeration. Ammonia is a toxic substance that is easy to identify in case of leakage, and that needs special handling care. Ammonia belongs to the safety group B1 i.e., a toxic non-flammable gas. It is a natural substance with no harmful effect to the ozone layer of the atmosphere and with zero global warming potential (GWP).

The used working fluids for ORC are non-toxic refrigerants, but the high values of their GWP make these products of environment concern. The EU F-Gas regulation imposes a decrease until 2030 on the average GWP of the refrigerants. From 2015 to 2030 the average GWP should drop from 2000 to 420, while it is 1260 in 2018. The most common working fluids for ORC are R245fa, a hydrofluorocarbon (HFC) with a GWP of 1030, and R245ca, which is also a HFC, with a GWP of 693. R245fa belongs to safety group B1, as well as ammonia, both causing some concern regarding the use in marine applications.

\subsection{Organic Rankine Cycle}

Organic Rankine cycle follows a Rankine cycle using a refrigerant as working fluid. Fig. 1 depicts a single stage ORC showing a pump (P) to maintain a high pressure in the lines 1 and 4, an evaporator (EVP) that receives heat from the exhaust gas, a turbine (ORCT), and a condenser (COND) that reject heat to a water stream. The ORCT expands the gas from the high-pressure line (1) to the low-pressure one (2), producing electricity in the generator $\mathrm{G}$.

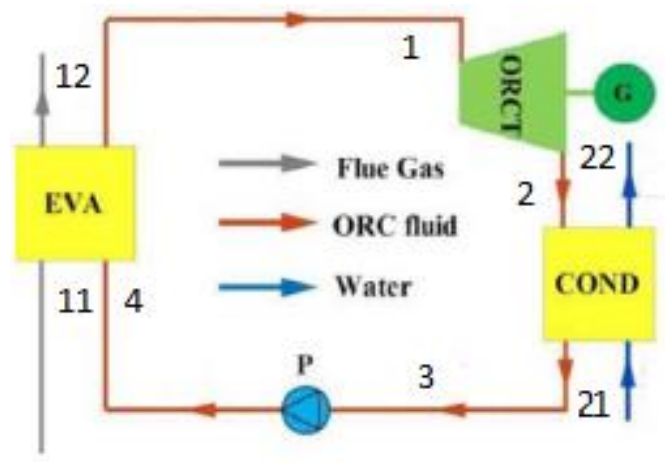

Fig. 1. ORC scheme (adapted from [6])

A variation of the single stage ORC uses a regenerator between the exit of the turbine and the entrance of the evaporator, reducing the heat released in the condenser. The temperature of the exhaust gases (11 to 12 ) determines the pressure on the evaporator, and the temperature of the condensing water at (21) determines the pressure on the condenser. Therefore, external conditions determine the behaviour of the system.

The coolant needs to be at the superheated vapour state at (1), in order to preserve the blades of the turbine from erosion. For R245fa, the pressure ratio in the turbine is about 20 , and the evaporator pressure is about 1 bar. The mass flow and the temperature of the exhaust gas are the key factors to define the power of the ORC equipment.

\subsection{Kalina Cycle}

Kalina cycle may have many schemes, the most common one is the KC11 as depicted in Fig. 2. A common variation of the flow diagram of $\mathrm{KC} 11$ puts the separator (SEP) before the evaporator (EVA). Most of the current industrial applications of $\mathrm{KC}$ use a mixture of ammonia and water. There are experimental works that use zeotropic mixtures of $\mathrm{HFC}$ to low the evaporating pressure.

In $\mathrm{KC}$, the turbine (AWT) needs a high concentration of ammonia because it produces more work per fluid flow unit than a lower concentration. The turbine has a pressure ratio of about 15 and drives the generator $(\mathrm{G})$. In the condenser (COND), it is desirable a higher temperature than in the turbine exit, as to simplify rejecting the heat. The absorber $(\mathrm{AB})$ makes the water to absorb the ammonia, therefore, increasing the temperature of the mixture. Due to the zeotropic behaviour of the ammonia-water, the boiling and condensing temperatures varies following the nonisothermal evolution of the fluids in the evaporator and the condenser.

Lines 1 and 2 have rich ammonia molar concentration (typically 95\%), lines 3, 4, 5, 6 and 7 have a lean concentration $(60 \%)$ and lines 8,9 and 10 a very poor concentration of ammonia (30\%). Pump (P) imposes a 
differential pressure between the low-pressure lines $(2$, 3, 4 and 10) and all the other high-pressure lines.

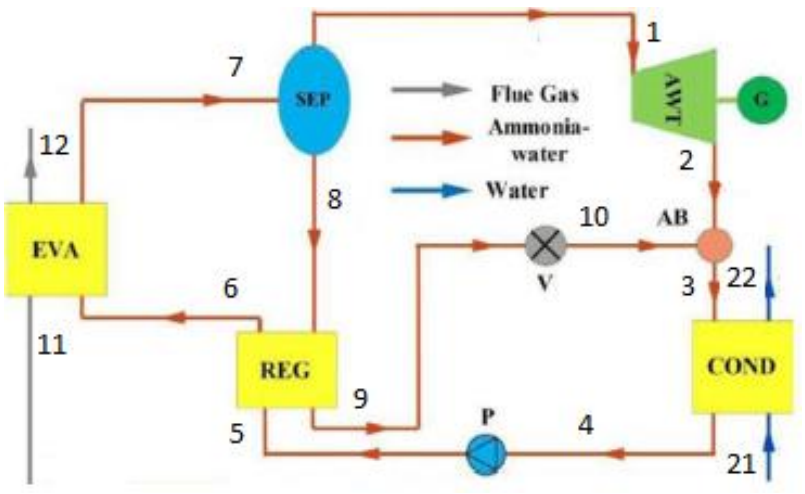

Fig. 2. KC11 scheme (adapted from [6])

The AWT and the valve (V) reduce the pressure from high to low. The regenerator (REG) allows recovering heat from the very poor concentration stream (8) to the evaporator stream.

The efficiency of the system is highly dependent on the concentration of ammonia in the AWT, therefore depending on the SEP and on the working mode of V. The valve $\mathrm{V}$ allows adjusting the amount of water rich solution that is required to correct the concentration of the ammonia rich solution. Thus, the efficiency of the $\mathrm{KC}$ mostly depends on the SEP and on the control of $\mathrm{V}$, as they determine the ammonia concentration entering the turbine and the condenser. The optimization of the different concentrations is a key factor regarding the efficiency of the system.

As in any other thermal machine, the efficiency of $\mathrm{KC}$ depends on temperature of the heat source and the temperature of the cold source.

\section{The AD evaluation}

The highest-level functional requirement (FR) of the designs under evaluation is:

$F R_{0}$ - Produce work from waste heat,

and the corresponding DP is:

$D P_{0^{-}}$Thermal engine.

For a thermal engine, the second law of Thermodynamics states that a cycled device needs to receive heat from a high temperature sink and reject heat to a low temperature sink in order to produce work. Moreover, the engine needs a working fluid to work, making the first level FRs of a thermal engine to be:

$F R_{1}$ - Receive heat;

$F R_{2}$ - Reject heat;

$F R_{3}$ - Produce work;

$F R_{4}$ - Transport thermal energy in cycle.

The next subsection reveals the DPs of each system, $\mathrm{ORC}$, and $\mathrm{KC}$, at the first and the second levels of decomposition.
AD classifies the designs as uncoupled, decoupled and coupled. The former are the best, and the latter are the poorest [8]. The FRs are actions that are formally defined in an independent way [9]. Moreover, FRs need to be cumulative exhaustive and mutually exclusive [10], which is a condition to attain during the decomposition process. At each level of decomposition, one may start by defining the nominal working conditions that allow defining the design matrix [11].

\subsection{AD application to ORC}

The ORC into consideration has R245fa as working fluid to transport thermal energy, uses the EVA to receive heat, COND to reject the heat, and the set of turbomachinery, $\mathrm{P}$ and ORCT, to produce work. Therefore the DPs are:

$$
\begin{aligned}
& D P_{1}-\text { EVA } \\
& D P_{2}-\text { COND } \\
& D P_{3} \text { Turbomachinery } \\
& D P_{4}-\text { R245fa }
\end{aligned}
$$

Equation 1 depicts the design equation of the ORC at the first level of decomposition showing that it is a decoupled design. Selecting a specific working fluid, $\mathrm{DP}_{4}$, makes it an 3-FR, 3-DP uncoupled design.

$$
\left[\begin{array}{l}
F R_{1} \\
F R_{2} \\
F R_{3} \\
F R_{4}
\end{array}\right]=\left[\begin{array}{llll}
X & & & X \\
& X & & X \\
& & X & X \\
& & & X
\end{array}\right] \cdot\left[\begin{array}{c}
D P_{1} \\
D P_{2} \\
D P_{3} \\
D P_{4}
\end{array}\right]
$$

\begin{tabular}{|c|c|}
\hline FR & DP \\
\hline$F R_{11}$ : Recover wasted heat & $D P_{11}$ : Exhaust gas \\
\hline $\begin{aligned} F R_{12}: & \text { Transfer high } \\
& \text { temperature heat }\end{aligned}$ & $\begin{array}{l}D P_{12}: \text { Heat exchanger } \\
\text { of EVA }\end{array}$ \\
\hline $\begin{array}{l}F R_{21}: \text { Choose low temperature } \\
\text { storage sink }\end{array}$ & $\begin{aligned} & D P_{21}: \text { Sea water } \\
& \text { supply system }\end{aligned}$ \\
\hline $\begin{aligned} F R_{22}: & \text { Transfer low } \\
& \text { temperature heat }\end{aligned}$ & $\begin{aligned} D P_{22}: & \text { Heat exchanger } \\
& \text { of COND }\end{aligned}$ \\
\hline $\begin{aligned} F R_{31}: \text { Maintain a pressure } \\
\text { differential }\end{aligned}$ & $D P_{31}:$ Pump \\
\hline$F R_{32}$ : Perform fluid expansion & $D P_{32}:$ ORCT \\
\hline
\end{tabular}

Therefore, $F_{4}$ turns to be a leaf and the decomposition proceeds with the other FRs.

Table 1 shows the second level of decomposition.

Table 1. The second level of decomposition for ORC.

On the second level of decomposition, new relationships appear, expressed on a new design matrix.

The ORC engine works in cycle so that making any change in any component would affect all the others. The design of the system will be considered in nominal conditions, although one should notice the impact of the external conditions in the design matrix. 
Transferring heat at high temperature depends on the temperature and mass of the exhaust gas, in the same way, that the sea water system influences the transfer of heat at low temperature. Moreover, defining the temperatures of source and sink reservoirs fix the working pressures of the refrigerant. Therefore, maintaining the pressure differential in P and ORCT is a function of the exhaust gas and the seawater temperatures.

Equation 2 shows the design matrix on the second level of decomposition. In order to compare systems, external conditions are set, fixing the temperature of exhaust gases and the sea water at nominal conditions. On Equation 2, the influence of external condition are set with a lowercase " $x$ " standing for a minor influence. Setting values for the external conditions, then the design is an uncoupled design.

$$
\left[\begin{array}{l}
F R_{11} \\
F R_{12} \\
F R_{21} \\
F R_{22} \\
F R_{31} \\
F R_{32}
\end{array}\right]=\left[\begin{array}{cccccc}
X & & & & & \\
x & X & & & & \\
& & X & & & \\
& & x & X & & \\
x & & x & & X & \\
x & & x & & & X
\end{array}\right] \cdot\left[\begin{array}{l}
D P_{11} \\
D P_{12} \\
D P_{21} \\
D P_{22} \\
D P_{31} \\
D P_{32}
\end{array}\right]
$$

\subsection{AD application to KC}

The first level of decomposition for the $\mathrm{KC}$ is similar to the ORC. The difference is just the working fluid. Therefore, the DPs are the following:

$$
\begin{aligned}
& D P_{1}-\text { EVA } \\
& D P_{2} \text { COND } \\
& D P_{3} \text { Turbomachinery } \\
& D P_{4}-\text { Ammonia-water }
\end{aligned}
$$

Equation 1 also applies to $\mathrm{KC}$, as well as the considerations regarding the working fluid. $F R_{4}$ turns a leave, and the decomposition of the $\mathrm{KC}$ design proceeds with the other three FRs. According to the aforesaid on section 2.2, new internal relationships are expected for this system on the second level of decomposition.

Again, the exhaust gas impacts the heat transfer at high temperature. In addition, the seawater system affects the heat transfer at low temperature. At last, the temperatures of the exhaust gas and the seawater define the temperatures of the ammonia-water at the evaporator and condenser. Furthermore, Fig. 2 introduces a regenerator that has a positive impact on the needs of heat at high temperature.

The equations of state of ammonia-water make enthalpy and pressure to change according to the concentration of ammonia for a given temperature.

Table 2 shows the FRs and DPs for $\mathrm{KC}$ system on the

\begin{tabular}{|c|c|}
\hline FR & DP \\
\hline$F R_{11}$ : Recover wasted heat & $D P_{11}$ : Exhaust gas \\
\hline $\begin{array}{l}F R_{12}: \text { Transfer high temperature } \\
\text { heat }\end{array}$ & $\begin{aligned} & D P_{12}: \text { Heat } \\
& \text { exchanger of } \\
& \text { EVA } \\
&\end{aligned}$ \\
\hline$F R_{13}$ : Recover internal heat & $D P_{22}:$ REG \\
\hline $\begin{array}{l}F R_{21}: \text { Choose low temperature } \\
\text { storage sink }\end{array}$ & $\begin{array}{l}D P_{21}: \text { Sea water } \\
\text { supply system }\end{array}$ \\
\hline $\begin{array}{l}F R_{22}: \text { Transfer low temperature } \\
\text { heat }\end{array}$ & $\begin{array}{l}D P_{22}: \text { Heat exchanger } \\
\text { of COND }\end{array}$ \\
\hline $\begin{array}{c}F R_{23}: \begin{array}{l}\text { Reduce ammonia } \\
\text { concentration }\end{array}\end{array}$ & $D P_{23}:$ Set $\mathrm{V}+\mathrm{AB}$ \\
\hline $\begin{array}{l}F R_{31}: \text { Maintain a pressure } \\
\text { differential }\end{array}$ & $D P_{31}:$ Pump \\
\hline$F R_{32}:$ Perform fluid expansion & $D P_{32}: \mathrm{AWT}$ \\
\hline $\begin{array}{c}F R_{33}: \text { Increase ammonia } \\
\text { concentration }\end{array}$ & $D P_{33}: \mathrm{SEP}$ \\
\hline
\end{tabular}
second level of decomposition. It is worth to notice the introduction of new FRs regarding the changing of ammonia concentration along the thermodynamic cycle.
Table 2. The second level of decomposition of KC.

Therefore, the role of the valve control and the absorber to reduce the ammonia concentration in the condenser; as well as the role of the separator to increase the ammonia fraction in the turbine, strongly influence the behavior of the system. A trade-off must be made between the performance regarding the fluid expansion vs. the transfer of heat at low temperature, which affects each other. Moreover, the lean concentration of ammonia in line 7 (Fig. 2) affects the behavior of the evaporator.

The set, valve and absorber, plays a special role by influencing the transfer at low temperature in the condenser, and by changing the performance of fluid expansion in the turbine.

Equation 3 shows that $\mathrm{KC}$ is a coupled design, despite fixing the external temperatures at the nominal conditions.

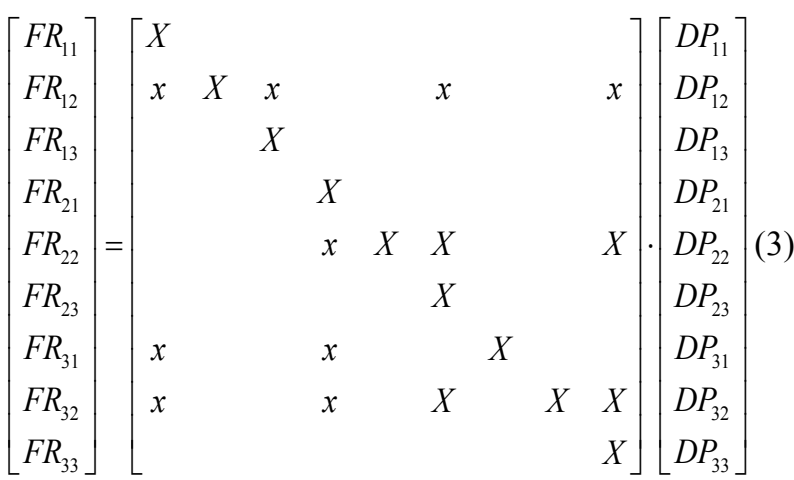

The most important relationships are set at Equation 3 by a " $X$ ", meaning they have a larger absolute value than all other off-diagonals. This decomposition is in accordance with a corollary of Theorem 8 of $\mathrm{AD}$ that allows neglecting low order $\partial F R / \partial D P$. Therefore, it is possible a design to be an uncoupled design at the first level of decomposition and coupled at the second level of decomposition. 


\section{Discussion}

The efficiency of the ORC and the $\mathrm{KC}$ are similar in what concerns to the recovery of heat from the exhaust gas of a Diesel engine. Common installations of ORC use R245fa, which is a hazardous, toxic and nonflammable fluid of B1security class, which belongs to the same group of the ammonia fluid that is used in the $\mathrm{KC}$ systems. Anyway, ammonia KC systems need about 100 bar pressure to work, thus requiring special components. Moreover, the AWT turbine should be multistage or should rotate at high speed to have a reasonable isentropic efficiency, due to the head loss. Some concerns may arise regarding the corrosion caused by the ammonia-water mixture, but the market has already a strong experience on choosing steels to work with the ammonia fluid.

In what regards to this paper, we found that fixing the external conditions, then $\mathrm{KC}$ is a coupled design and ORC is uncoupled.

Considering the external conditions, at nominal conditions, ORC is a decoupled design, and $\mathrm{KC}$ is a coupled design.

According to $\mathrm{AD}$, a good design needs to be uncoupled or decoupled. A coupled design is a poor design, having a lower probability of success. If it is possible to achieve two uncoupled, or two decoupled designs, then the design team must apply the information axiom and choose the design with lower information. The classification of the design may change during decomposition, being necessary to compare designs at the same level of decomposition.

Based on the application of the AD theory, the ORC is the design to choose, because it is a better design than the $\mathrm{KC}$ design.

\section{Conclusions}

This paper applies Axiomatic Design (AD) to compare the use of organic Rankine cycle (ORC) and Kalina cycle (KC) for recovering heat from the exhaust gas of a marine Diesel engine.

ORC and $\mathrm{KC}$ use hazardous working fluids that require special caution regarding the installation on marine vessels. Both cycles can achieve similar efficiencies, although the $\mathrm{KC}$ needs higher evaporating pressure and more technical concerns than for the ORC systems.

$\mathrm{AD}$ classifies $\mathrm{KC}$ as a coupled design and $\mathrm{ORC}$ as an uncoupled design. As a result, $\mathrm{KC}$ needs optimization in order to achieve the efficiency of the ORC, thus needing a special control of the valve $\mathrm{V}$.

As a conclusion, according to the $\mathrm{AD}$ theory, the ORC system is a better design than the $\mathrm{KC}$ system. This result is in accordance to many technical opinions regarding this subject.

The authors gratefully thank the sponsorship of Fundação para a Ciência e Tecnologia through the Strategic Project UID/EMS/00667/2013 - UNIDEMI.

\section{References}

1. World Resources Institute, CAIT Climate Data Explorer, www.wri.org/blog/2017/04/interactivechart-explains-worlds-top-10-emitters-and-howtheyve-changed, accessed May 20, 2018

2. https://europa.eu/european-union/topics/energy-en, accessed June 4, 2018

3. D. V. Singh, E. Pedersen, A review of waste heat recovery technologies for maritime applications, Energy Conversion and Management, 111, 315-328 (2016)

4. U. Larsen, O. Sigthorsson, F. Haglind, A comparison of advanced heat recovery power cycles in a combined cycle for large ships, Energy, 74, 260-268 (2014)

5. P. Bombarda, C. M. Invernizzi, C. Pietra, Heat recovery from Diesel engines: A thermodynamic comparison between Kalina and ORC cycles, Applied Thermal Engineering, 30, 212-219 (2010)

6. A. Nemati, H. Nami, F. Ranjbar, M. Yari, A comparative thermodynamic analysis of ORC and Kalina cycles for waste heat recovery: A case study for CGAM cogeneration system, Case Studies in Thermal Engineering, 9, 1-13 (2017)

7. D. Lin, Q. Zhu, X. Li, Thermodynamic comparative analyses between (organic) Rankine cycle and Kalina cycle, The 7th Int. Conf. on Applied Energy ICAE2015, Energy Procedia, 75, 1618-1623 (2015)

8. N.P. Suh, The principles of design, Oxford Univ. Press, N.Y., (1990)

9. M. K. Thompson, A Classification of Procedural errors in the definition of functional requirements in axiomatic design in Axiomatic Design Theory, Proc. 7th Int. Conf. on Axiomatic Design (ICAD 2013), Worcester, USA, (2013)

10. C. A. Brown, R. Henley, Metrics for Developing Functional Requirements and Selecting Design Parameters in Axiomatic Design, Procedia CIRP, 53, 113-118, (2016)

11. M. Cavique, J. Fradinho, A. Gabriel-Santos, A. Gonçalves-Coelho and A. Mourão, The Iterative Nature of the "Zig" and How to Define the "Hows", 11 th Int. Conf. on Axiomatic Design (ICAD 2017), MATEC Web of Conferences, 127, 01007 (2017) 